\title{
Posicionamiento laboral de los egresados de la carrera de licenciatura en administración de FACEM-UNICAN de Salto del Guairá, 2021
}

\author{
Derlis Daniel Duarte Sánchez \\ derlisduarte@facem.edu.py \\ https://orcid.org/0000-0002-6717-2873. \\ Doctorando en Contabilidad y Auditoría. \\ Universidad Nacional de Canindeyú-Paraguay
}

\section{RESUMEN}

El objetivo del presente estudio fue analizar el posicionamiento laboral de los egresados de la carrera de Licenciatura en Administración de la Facultad de Ciencias Económicas y Empresariales de la Universidad Nacional de Canindeyú de Salto del Guairá en el año 2021. Se utilizó una metodología de enfoque cuantitativo, de diseño no experimental, de nivel descriptivo, de corte transversal, la técnica de recolección de datos fue la encuesta, se utilizó el muestreo aleatorio simple, se realizó distintas operaciones donde fueron sometidos los datos (clasificación, registro, tabulación y análisis) para descifrar lo que revelan los datos recogidos. La población del estudio fueron los egresados de los años 2019 y 2020. Como resultado se obtiene que el 95\% de los egresados de la carrera de Licenciatura en Administración cuentan con empleo, al mismo tiempo que el 27.3\% de sexo masculino y el $72,7 \%$ son de sexo femenino por lo que se destaca la participación de mujeres en la profesión universitaria. En este mismo contexto, el 81\% trabajan en el sector terciario por el lugar estratégico comercial que se encuentra la ciudad porque abundan los grandes comercios. Además, se resalta que el $65 \%$ han sido promovidos del puesto de trabajo como resultado de su preparación académica porque el $90.5 \%$ ya contaban con empleos. Se confirma que la formación Universitaria ayuda a encontrar empleos y en ascender en los puestos de trabajos en la ciudad de Salto del Guairá en el año 2021.

Palabras claves: formación universitaria; posicionamiento laboral; participación femenina; ascenso laboral; satisfacción académica. 


\title{
Labor positioning of the graduates of the bachelor's degree in administration from FACEM-UNICAN of Salto del Guairá, 2021
}

\begin{abstract}
The objective of this study was to analyze the job positioning of graduates of the Bachelor of Administration degree from the Faculty of Economic and Business Sciences of the National University of Canindeyú de Salto del Guairá in the year 2021. A quantitative approach methodology was used, non-experimental design, descriptive level, cross-sectional, the data collection technique was the survey, simple random sampling was used, different operations were carried out where the data were submitted (classification, registration, tabulation and analysis) to decipher what the collected data reveals. The study population was the graduates of the years 2019 and 2020. As a result, it is obtained that $95 \%$ of the graduates of the Bachelor of Administration career have a job, at the same time as $27.3 \%$ of males and $72,7 \%$ are female, which is why the participation of women in the university profession stands out. In this same context, $81 \%$ work in the tertiary sector due to the strategic commercial location of the city because there are many large stores. In addition, it is highlighted that $65 \%$ have been promoted from the job as a result of their academic preparation because $90.5 \%$ already had jobs. It is confirmed that university training helps to find jobs and rise in jobs in the city of Salto del Guairá in the year 2021.
\end{abstract}

Keywords: university education; job positioning; female participation; job advancement; academic satisfaction.

Artículo recibido: 20 diciembre. 2021 Aceptado para publicación: 10 enero 2022 Correspondencia: derlisduarte@ facem.edu.py Conflictos de Interés: Ninguna que declarar 


\section{INTRODUCCIÓN}

La inversión en las universidades es indispensable para el buen desarrollo de un país.

Sin una sólida infraestructura de Educación Superior, que tiene su sustento en la Universidad, el crecimiento nacional y la calidad de vida están condenados al fracaso (Lugo et al., 2017).

La educación permite optar a mejores salarios, se muestra que, a más años de escolaridad, mejor es el salario por hora para los jóvenes. De acuerdo a este análisis, acceder a una educación superior duplica la expectativa a nivel regional de ingresos por hora (Serafini \& Bonilla, 2016).

Por otra parte, desde el inicio de la era moderna, la inserción laboral de los jóvenes es el elemento clave para pasar a la vida adulta, dado que los ingresos propios generan la base material para disminuir y luego eliminar la dependencia económica respecto de los padres y establecer un hogar propio (Weller, 2007, p. 2).

La inserción laboral se relaciona con el porcentaje de avance en dos diferentes metas: la formación y el sistema económico. Por un lado, se necesita un razonable equilibrio entre los criterios de formación académica (conocimientos, competencias y actitudes) y los requerimientos del trabajo. En conjunto, unos y otros son elementos necesarios para desarrollarse exitosamente en el campo laboral. Por el otro, es necesario equilibrar el número de egresados de las instituciones de educación superior y la capacidad de absorción del sistema económico en condiciones laborales apropiadas para sus perfiles profesionales (Pérez Cruz \& Pinto Pérez, 2020, p. 5).

Por consiguiente, conocer las ocupaciones y las condiciones de trabajo que realmente tienen los egresados de carreras universitarias es un tema particularmente importante, pues por lo general se parte en forma acrítica del supuesto de que la simple posesión de un título universitario, le permite a su poseedor trabajar como profesionista y ubicarse en la parte alta de la pirámide ocupacional, donde se reciben las mejores remuneraciones (Rodríguez Solera, 2000, p. 5).

Por lo anterior, la investigación nace desde el interés del investigador en conocer el posicionamiento laboral de los egresados de la carrera de Licenciatura en Administración, si los mismos están con empleo, que tipo de trabajo tienen, si están cursando algún postgrado para obtener datos estadísticos y de esa manera poder 
actualizar las informaciones de los egresados de la Facultad de Ciencias Económicas y Empresariales de la Universidad Nacional de Canindeyú de Salto del Guairá-Paraguay.

\section{MATERIALES Y METODOS}

El enfoque de la investigación es cuantitativo porque los resultados se reflejan en cantidades numéricas (Hernandez Sampieri \& Mendoza Torres, 2018, p. 6). El diseño de investigación es no experimental porque no se realiza ningún tipo de experimento (Hernandez Sampieri \& Mendoza Torres, 2018, p. 174), además, el nivel de investigación es descriptivo porque se describe características importantes de los egresados de la carrera de Licenciatura en Administración (Peña Marroquín, 2018, p. 4). Por tanto, el tiempo de la investigación es de corte trasversal porque la recolección de datos se realiza en un momento dado (Müggenburg \& Pérez, 2018, p. 4). La modalidad es de campo porque para la recolección de datos se realiza a través en el lugar de los hechos (Peña Marroquín, 2018, p. 20).

En la presente investigación como instrumento de recolección de datos se utilizó el cuestionario, la misma se ha diseñado de acuerdo a la problemática, se ha utilizado el formulario de google con preguntas cerradas facilitando al encuestado a que responda a través de la comodidad de su teléfono, todos en la modalidad de selección múltiple.

La técnica de recolección de datos fue la encuesta, la misma fue diseñada conforme a la problemática, se diseñaron las preguntas de la investigación con la finalidad de alcanzar el objetivo general. Para la aplicación del instrumento se seleccionó la herramienta de Google Forms, la cual permite realizar cuestionarios vía on line.

La población de la investigación está compuesta por los egresados de la carrera de Licenciatura en Administración de los periodos académicos 2019 y 2020 de la ciudad de Salto del Guairá que son 35 egresados en total, de los cuales se pudo encuestar a 22 egresados ya que algunos han migrado a otros lugares y no se les pudo contactar en el tiempo de la investigación. En la presente investigación se utilizó el muestreo aleatorio simple donde a cada elemento de la población y a cada posible muestra de un tamaño determinado, tiene la misma posibilidad de ser seleccionado (Otzen \& Manterola, 2017).

\section{RESULTADOS y DISCUCIÓN}

Con base en los resultados obtenidos el $72.7 \%$ de la población encuestada son de sexo femenino y el $27.3 \%$ de sexo masculino, por consiguiente, en las últimas décadas se ha 
producido un rápido aumento del nivel de estudios superiores en todo el mundo. La inversión de la brecha de género en la educación se produjo de la mano del considerable aumento del nivel educativo. Gran parte de este crecimiento se debe al aumento del nivel educativo de las mujeres (Organización de las Naciones Unidas para la Educación, la Ciencia y la Cultura \& Instituto Internacional para la Educación Superior en América Latina y el Caribe, 2021, p. 14).

Además, es importante resaltar que los datos económicos muestran que dar a las mujeres las mismas oportunidades que a los hombres mejora la competitividad y productividad de un país, lo que a su vez incide positivamente en el crecimiento económico y en la reducción de la pobreza. A escala mundial, la eliminación de todas las formas de discriminación contra la mujer permitiría aumentar la productividad per cápita un 40\%, según el Banco Mundial (informe Doing Business 2017), publicado por (Organización Mundial del Comercio, 2017).

Por otra parte, el $95.5 \%$ de los egresados cuentan con empleo y el $4.5 \%$ no cuenta con empleo. Según Cancelo (2010) la vinculación entre la educación superior y el empleo pueden describirse como parte de una meritocracia educacional. Lo ideal es que los estudiantes tengan la oportunidad de tener éxito, desde el punto de vista educativo, sea cual sea su procedencia socio biográfica; cuanto mayor es el nivel alcanzado por la persona al final de su educación previa al trabajo, más probabilidades tendrá de éxito en el mundo laboral, aceptando las dimensiones de lo que generalmente se considera un trabajo o situación en la sociedad deseable, es decir, un buen sueldo, estatus, poder e influencia social (Álvarez Gavilanes \& Fernández, 2015, p. 5).

Por lo anterior se puede confirmar que la Facultad de Ciencias Económicas y Empresariales de la Universidad Nacional de Canindeyú mantiene un vínculo fuerte con el mundo laboral ya que el $95.5 \%$ de los estudiantes cuentan con empleo.

El hecho de contar con un título universitario o el estar cursando los últimos años de alguna carrera son variables importantes para obtener un puesto de trabajo. Existen demandas específicas en cuanto a formación académica, relacionadas al cargo y al tipo de organización (Miranda Espinoza, 2016, p. 25).

Además, en relación al tiempo de encontrar trabajo después de egresar el $90.5 \%$ de la población ya contaba con empleos, esto indica que siendo estudiantes universitarios si ya estaban trabajando y el $9.5 \%$ han mencionado que les ha llevado más de un año para 
encontrar empleo después de graduarse. La inserción laboral, por tanto, se ha convertido en uno de los principales objetivos a conseguir con la realización de prácticas en empresas, esto afirma que los alumnos que ya cuentan con empleo, van ganando experiencia laboral que muchos optan por realizar su pasantía profesional en el lugar trabajo (Martínez Martín, 2003, p. 5).

La teoría del capital humano, desarrollada por Becker en 1964, destaca formación académica y la formación continua como la principal inversión que realizan los individuos para incrementar su productividad (Salas Durazo \& Murillo García, 2013). Aquí la teoría afirma que la formación es el principal inversión de un individuo para incrementar su productividad.

Asimismo, las causas de no contar con empleo se obtiene que el 33.3\% han mencionado porque algunas labores no han cumplido con sus expectativas, por otra parte, el 33,3\% no pudo encontrar empleo porque aún no le ha llegado el título, cabe resaltar que la gestión del título en el Ministerio de Educación y Ciencias para su registro tarda aproximadamente 6 meses y por último el $33.3 \%$ no pudo encontrar empleo por la falta de experiencias laborales.

El desempleo es un problema tanto económico como social para los jóvenes, ya que económicamente se desperdician muchos recursos al tener a jóvenes universitarios con amplios conocimientos y que estén desocupados. Esto es perjudicial para ellos mismos, para su familia y para el país en general (Guillén Moreno et al., 2021).

$\mathrm{Al}$ respecto de las actividades de dedicación de los egresados, los resultados arrojan que el $4.7 \%$ están en el sector primario (extractivo, agricultura, pesca, ganadería, minería), el $14.3 \%$ en el sector secundario y el $81 \%$ en el sector terciario (servicio, comercio, educativo, gobierno, salud), es importante destacar que la ciudad de Salto del Guairá abundan los grandes comercios como shopines (Grandes comercios), supermercados y más, es por la posición estratégica en donde se encuentra con la frontera con el Brasil.

El comercio es una herramienta importante para el desarrollo y sabemos que sin él, el crecimiento, la creación de puestos de trabajo y el desarrollo son más difíciles de lograr (Organización Mundial del Comercio, 2022).

Por tanto, el $65 \%$ de la población encuestada ha indicado que han sido promovidos del puesto y el 35\% han mantenido sus puestos laborales. Carrera, Parreño, \& Ayala (2018), afirma que el desempeño profesional hace referencia a todas las acciones llevadas a 
cabo por una persona que aprendió una profesión u oficio, que se puede dar por un proceso formativo o basado en la experiencia; tal desempeño debe mostrar habilidades, conocimientos e idoneidad en la realización de labores, estén estas vinculadas a la producción de bienes o prestación de servicios (Auccapuri et al., 2021, p. 11).

En ese mismo contexto sobre el nivel de consideración sobre la respuesta de la preparación académica en frente a las exigencias laborales se obtiene que el $27.3 \%$ mencionan que la preparación ha sido excelente, el 50\% menciona que ha sido muy buena y el $22.7 \%$ ha sido bueno.

Con base a lo mencionado, es prudente resaltar que una profesión no siempre se adquirirá de manera técnica, científica; sino que este proceso puede darse por un aprendizaje apoyado en la experiencia; en ambos casos el aspecto relevante es que en el cumplimiento de las funciones encomendadas el desempeño profesional de una persona esté acorde a los requerimientos de la organización y a las exigencias de la sociedad (Auccapuri et al., 2021, p. 11).

Por otra parte, el personal docente en las instituciones de educación superior (IES) tiene la tarea de transmitir conocimientos a sus alumnos, ayudarles a desarrollar las competencias que necesitan para la vida y el trabajo y crear nuevo conocimiento a través de publicaciones científicas (Gómez, 2019). Además, las sociedades desarrolladas tienden hacia una economía basada en el conocimiento, en donde la capacidad para crear cuenta más que los tradicionales factores de producción como fuente de desarrollo y de creación de riqueza. Es ahí donde la educación juega un papel fundamental y, dentro de ella, la educación superior y su relación con el mercado de trabajo cobra cada vez mayor importancia (Salgado Vega, 2005).

\section{CONCLUSIÓN}

Habiendo realizado los procesos de investigación, se logró conocer que el 95\% de los egresados de la carrera de Licenciatura en Administración cuentan con empleo, al mismo tiempo que el $72,7 \%$ son de sexo femenino por lo que se destaca la participación de mujeres la profesión universitaria. En este mismo contexto, el $81 \%$ trabajan en el sector terciario por el lugar estratégico comercial que se encuentra la ciudad porque abundan los grandes comercios. 
Además, se resalta que el $65 \%$ han sido promovidos del puesto de trabajo como resultado de su preparación académica porque el $90.5 \%$ ya contaban con empleos. Por consiguiente, se destaca sobre el nivel de satisfacción sobre la preparación académica en frente a las exigencias del mercado, que el 27.3\% mencionan que la preparación ha sido excelente, el $50 \%$ menciona que ha sido muy buena y el $22.7 \%$ ha sido bueno.

Por todo lo expuesto, esto indica que en la Universidad Nacional de Canindeyú en la Facultad de Ciencias Económicas y Empresariales de la carrera de Licenciatura en Administración se trabaja de forma excelente con calidad resaltando que es una carrera acreditada por la Agencia Nacional de Evaluación y Acreditación de la Educación Superior (ANEAES) es el organismo técnico encargado de evaluar y acreditar la calidad académica de los Institutos de Educación Superior (Facultad de Ciencias Económicas y Empresariales de la Universidad Nacional de Canindeyú, 2018).

\section{REFERENCIAS BIBLIOGRÁFICAS}

Álvarez Gavilanes, J. E. Á., \& Fernández, A. R. (2015). La empleabilidad de graduados universitarios en el contexto latinoamericano. Realidades de uniande. 16. https://www.redalyc.org/pdf/4780/478047208001.pdf

Auccapuri, A. A. H., Ortiz, Z. L., Mendoza, M. U. U., Salas, J. C., \& Palacios, M. D. V. (2021). Planificación curricular en la enseñanza universitaria y desempeño profesional de egresados en educación. Ciencia Latina Revista Científica Multidisciplinar, $5(3)$, 2563-2589. https://ciencialatina.org/index.php/cienciala/article/view/474

Facultad de Ciencias Económicas y Empresariales de la Universidad Nacional de Canindeyú. (2018). Acreditación de la carrera de Licenciatura en Administración por la ANEAES. http://www.facem.edu.py/index.php/11noticias/200-acreditaci\%C3\%B3n-de-la-carrera-de-licenciatura-enadministraci\%C3\%B3n-por-la-aneaes

Gómez, E. R. D. (2019). Educación para la empleabilidad: Enfoque de la investigación educativa. IE Revista de Investigación Educativa de la REDIECH, 10(19), 121138. https://www.redalyc.org/journal/5216/521658239014/html/

Guillén Moreno, C. N., Blandón López, H. O., López Tórrez, K. A., \& Gómez García, R. (2021). Situación del desempleo en los jóvenes universitarios de Ciencias 
Económicas de la UNAN Managua / FAREM Estelí. Revista Multi-Ensayos, 30-38. https://doi.org/10.5377/multiensayos.v7i2.12155

Hernandez Sampieri, R., \& Mendoza Torres, C. P. (2018). Metodología de la investigación. Mc Graw Hill. 978-1-4562-6096-5

Lugo, A. J. D., Ávila, A. E. S., Peña, A. C., Moreno, M. A. B., \& González, D. I. R. (2017). Investigación sobre las oportunidades de empleo para los profesionistas recién egresados utilizando BSC. RIDE Revista Iberoamericana para la Investigación y el Desarrollo Educativo, 8(15), 116-134. https://doi.org/10.23913/ride.v8i15.293

Martínez Martín, R. (2003). La inserción laboral de los universitarios a través de las prácticas en $\quad$ empresas. 229. https://www.redalyc.org/pdf/997/99717912008.pdf

Miranda Espinoza, M. de dos A. (2016). Egresados de la facultad de Ciencias Económicas (UNA), y su incorporación al mercado laboral del sector público de la administración central período: 2003-2013 [Universidad Nacional de Asunción].

https://www.eco.una.py/eco/postgrado/tesis/2016/Maria_de_los_Angeles_Mira nda_espinoza_110416.pdf

Müggenburg, M. C., \& Pérez, I. (2018). Tipos de estudio en el enfoque de investigación cuantitativa. Enfermería Universitaria, 4(1). https://www.redalyc.org/pdf/3587/358741821004.pdf

Organización de las Naciones Unidas para la Educación, la Ciencia y la Cultura, \& Instituto Internacional para la Educación Superior en América Latina y el Caribe. (2021). Mujeres en la educación superior: ¿la ventaja femenina ha puesto fin a las desigualdades de género? (UNESCO). https://www.iesalc.unesco.org/wp-content/uploads/2021/03/Las-mujeres-en-laeducacio\%CC\%81n-superior_12-03-21.pdf

Organización Mundial del Comercio. (2017). Las mujeres y el comercio. OMC. https://www.wto.org/spanish/tratop_s/womenandtrade_s/womenandtrade_s.htm Organización Mundial del Comercio. (2022). Estimular el crecimiento económico y el empleo. https://www.wto.org/spanish/thewto_s/whatis_s/10thi_s/10thi03_s.htm 
Otzen, T., \& Manterola, C. (2017). Técnicas de Muestreo sobre una Población a Estudio. International Journal of Morphology, 35(1), 227-232. https://doi.org/10.4067/S0717-95022017000100037

Peña Marroquín, R. (2018). METODOLOGÍA DE LA INVESTIGACIÓN. 26. http://www.une.edu.pe/Sesion04-Metodologia_de_la_investigacion.pdf

Pérez Cruz, O. A., \& Pinto Pérez, R. (2020). Determinantes de la inserción laboral en egresados universitarios en México. RIDE Revista Iberoamericana para la Investigación $\quad y$ el Desarrollo Educativo, 11(21). https://www.ride.org.mx/index.php/RIDE/article/view/732

Rodríguez Solera, C. R. (2000). La inserción laboral de egresados de la educación $\begin{array}{lllll}\text { superior en el htado de hidalgo. } & 16 .\end{array}$ http://publicaciones.anuies.mx/pdfs/revista/Revista127_S2A1ES.pdf

Salas Durazo, I. A., \& Murillo García, F. (2013). Los profesionistas universitarios y el mercado laboral mexicano: Convergencias y asimetrías. Revista de la educación superior, 42(165), 63-81. http://www.scielo.org.mx/scielo.php?script=sci_abstract\&pid=S0185$\underline{27602013000100004 \& \operatorname{lng}=e s \& n r m=i s o \& \operatorname{lng}=e s}$

Salgado Vega, M. del C. (2005). Empleo y transición profesional en México. Papeles de población, 11(44), 255-285. http://www.scielo.org.mx/scielo.php?script=sci_abstract\&pid=S1405$\underline{74252005000200011 \& \operatorname{lng}=\mathrm{es} \& \mathrm{nrm}=\mathrm{iso} \& \ln \mathrm{ln}=\mathrm{es}}$

Serafini, V., \& Bonilla, M. I. (2016). Educación para la Vida y Formación para el Empleo. $\quad$ https://cien.org.gt/wpcontent/uploads/2017/03/Educacio\%cc\%81n-para-la-Vida-y-el-Empleo-vf.pdf

Weller, J. (2007). La inserción laboral de los jóvenes: Características, tensiones y desafíos. REVISTA DE LA CEPAL, 22. https://repositorio.cepal.org/bitstream/handle/11362/11192/092061082_es.pdf?s equence $=1 \&$ is Allowed $=\mathrm{y}$ 